\title{
On the effect of $\delta$-doping in self-switching diodes
}

\author{
A. Westlund, ${ }^{1}$ I. Iñiguez-de-la-Torre, ${ }^{2}$ P.-Å. Nilsson, ${ }^{1}$ T. González, ${ }^{2}$ J. Mateos, ${ }^{2}$ P. Sangaré, ${ }^{3}$ \\ G. Ducournau, ${ }^{3}$ C. Gaquière, ${ }^{3}$ L. Desplanque, ${ }^{3}$ X. Wallart, ${ }^{3}$ and J. Grahn ${ }^{1}$ \\ ${ }^{1}$ Department of Microtechnology and Nanoscience-MC2, Chalmers University of Technology, \\ SE-412 96 Gothenburg, Sweden \\ ${ }^{2}$ Departamento de Física Aplicada, Universidad de Salamanca, Plaza de la Merced s/n, 37008 Salamanca, \\ Spain \\ ${ }^{3}$ Institute of Electronics, Microelectronics and Nanotechnology, UMR CNRS 8520, University of Lille, \\ CS 60069, 59652 Villeneuve d'Ascq, France
}

(Received 17 July 2014; accepted 21 August 2014; published online 4 September 2014)

\begin{abstract}
Lowering the carrier concentration is presented as a way to considerably improve the performance of self-switching diode (SSD) detectors. A physics-based theoretical model was used to derive an expression for the responsivity of SSDs as a function of carrier concentration, mobility, and design parameters. Monte Carlo simulations confirmed the modeled effect of varying carrier concentration and channel width. SSDs were fabricated in InAs heterostructures with different $\delta$-doping levels. Radio frequency (RF) characterization at $50 \mathrm{GHz}$ reproduced the modeled trends. By reducing the carrier concentration in InAs SSDs with $40 \mathrm{~nm}$ wide channels from $2.7 \times 10^{12} \mathrm{~cm}^{-2}$ to $1.5 \times 10^{12} \mathrm{~cm}^{-2}(-44 \%)$, the noise equivalent power (NEP) improved from $130 \mathrm{pW} / \mathrm{Hz}^{1 / 2}$ to $87 \mathrm{pW} / \mathrm{Hz}^{1 / 2}$ (-33\%). C 2014 AIP Publishing LLC. [http://dx.doi.org/10.1063/1.4894806]
\end{abstract}

Self-switching diodes (SSDs) are semiconductor devices where the current in nanometer-sized channels is modulated by a field-effect. ${ }^{1}$ The nonlinear I-V relationship of the SSDs can be used for detection of millimeter-wave signals. ${ }^{2}$ SSDs have been utilized in mixers, ${ }^{3}$ however mainly as direct detectors, both with ${ }^{2,4}$ and without bias. ${ }^{5,6}$ The latter option is preferred when a low $1 / \mathrm{f} \mathrm{noise}^{7}$ is required, an important property for direct detection. Zero-bias detection at $1.5 \mathrm{THz}$ has been reported with GaAs SSDs. ${ }^{2}$ Previously, we demonstrated InAs SSDs with a responsivity of $34 \mathrm{~V} / \mathrm{W}$ and a noise-equivalent power (NEP) of $65 \mathrm{pw} / \mathrm{Hz}^{1 / 2}$ at $50 \mathrm{GHz}{ }^{6}$ In this study, we have investigated the influence of the $\delta$ doping level in InAs SSDs.

In most semiconductor devices, the doping level is a parameter that is fundamental to the device behavior. The influence of $\delta$-doping level on the performance of SSD detectors in terms of responsivity and NEP has not been reported, neither experimentally, nor theoretically, nor in device simulations. The effect of $\delta$-doping level in SSDs has only been studied in simulations, without experimental verification, and only with regard to I-V characteristics. ${ }^{8,9}$ In this study, InAs SSDs are fabricated in InAs/ $\mathrm{Al}_{80} \mathrm{Ga}_{20} \mathrm{Sb}$ heterostructures with different $\delta$-doping levels and electrically characterized. The $\delta$-doping mainly affects the sheet carrier concentration $n_{s}$ in the two-dimensional electron gas (2DEG) of the heterostructure. By modeling the SSD as a side-gated field-effect transistor (FET), ${ }^{10}$ a theoretical relationship between $n_{s}$, design and detector performance is derived. It is shown that reduction of the $\delta$-doping and thereby $n_{s}$, is beneficial for the performance of the InAs SSD detectors, both in terms of responsivity and NEP. Monte Carlo (MC) simulations and measurements confirm the model.

The design of an SSD channel is illustrated in Fig. 1. In this study, the number of channels in parallel were $N=43$. The channels were defined by trenches etched slightly below the two-dimensional electron gas (2DEG) of the heterostructure. ${ }^{11}$ The $\delta$-doping level and the channel width $W$ were the only studied design parameters. Other geometrical parameters were, as defined in Fig. 1: trench widths $W_{\mathrm{h}}=W_{\mathrm{v}}=100 \mathrm{~nm}$, channel length $L=1000 \mathrm{~nm}$, and channel separation $S=350 \mathrm{~nm}$. The 2DEG had a thickness $Z=15 \mathrm{~nm}$.

Fig. 1 also shows a simplified equivalent device circuit (extrinsic reactive elements were excluded) of the SSD. All electrical nonlinearity in the SSD is represented by the intrinsic non-linear resistance $R_{\mathrm{i}}$, which is modulated by the drain-connected flanges surrounding the channel. ${ }^{1}$ Other resistances defined in Fig. 1 constitute a parasitic linear resistance $R_{\mathrm{s}, \text { tot }}=R_{s 2}+R_{s a}+R_{d a}$.

The non-linearity of the SSD I-V translates directly into a detector responsivity. Considering $V_{D_{i}}$ as the voltage across $R_{i .}$, the intrinsic I-V curvature can be defined as $\gamma_{i}=\left(d^{2} I_{D} / d V_{D_{i}}^{2}\right) /\left(d I_{D} / d V_{D_{i}}\right)$. The voltage responsivity with lossless optimum radio frequency $(\mathrm{RF})$ match, $\beta_{\text {opt }}$, is then found as ${ }^{12}$

$$
\beta_{\text {opt }}=\frac{1}{2} R_{i} \gamma_{i} \times \frac{R_{i}}{R_{i}+R_{s, t o t}} .
$$

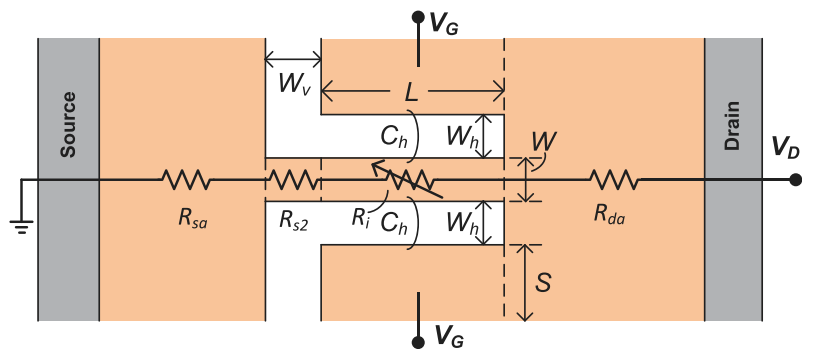

FIG. 1. Schematic top view of the geometry of one SSD channel, and an equivalent circuit. White fields depict etched tranches, reaching through the 2DEG. The fictional $V_{G}$ terminals are used in the analysis of the intrinsic SSD as a side-gated field-effect transistor. 
As seen from (1), a high $R_{i}$ increases $\beta_{\text {opt }}$. However, power transfer from an RF source with limited impedance to an SSD detector with large $R_{i}$ may become narrowband and lossy. To illustrate this trade-off, it is relevant to study the responsivity when the SSD is fed from a lower impedance, here $50 \Omega$, which is found as $\beta_{50 \Omega}=\beta_{\text {opt }}\left(1-|\Gamma|^{2}\right)$, where $\Gamma$ is the reflection coefficient from the source into the SSD detector.

The noise voltage across diode terminals at zero bias is the Johnson noise voltage $V_{n}=\sqrt{4 k T\left(R_{i}+R_{s, t o t}\right)}$ where $k$ is Boltzmann's constant and the physical temperature $T=290 \mathrm{~K}$, making the NEP with optimum power match

$$
N E P_{o p t}=\sqrt{4 k T\left(R_{i}+R_{s, t o t}\right)} / \beta_{o p t} .
$$

The NEP with a $50 \Omega$ source can be found as $N E P_{50 \Omega}=\sqrt{4 k T\left(R_{i}+R_{s, t o t}\right)} / \beta_{50 \Omega}$. A model for how $\delta$ doping and design affects the device performance can be derived by extending the direct current (DC) analysis of the intrinsic SSD as a double side-gated transistor. ${ }^{1,10}$ Referring to Fig. 1, the intrinsic SSD consists of $R_{i}$ and the two flanges acting as gates with potential $V_{G}$. The gates couple to the channel with the capacitance $2 C_{h}$ per unit channel length. It is assumed that no surface charge is present at the channel sidewalls, that the long channel approximation is valid and that the electron mobility $\mu_{n}$ is field-independent $\left(V_{D_{i}} \approx 0 \mathrm{~V}\right)$. Further, the SSD is assumed to be in depletion mode, i.e., $V_{D_{i}}>V_{T}$, where $V_{T}$ is a threshold voltage. Defining $V_{G}$ as the voltage of the flanges surrounding the channel, the drain current becomes ${ }^{10}$

$$
I_{D}=N K\left[\left(\frac{q n_{s} W}{2 C_{h}}+V_{G}\right) V_{D_{i}}-\frac{V_{D_{i}}^{2}}{2}\right],
$$

where $q$ is the elementary charge and $K=2 \mu_{n} C_{h} / L$. By setting $V_{G}=V_{D_{i}}$ and defining the threshold voltage as $V_{T}=-q n_{s} W / 2 C_{h}$, (3) transforms into

$$
I_{D}=N K\left(\frac{V_{D_{i}}}{2}-V_{T}\right) V_{D_{i}}
$$

from which $R_{i}$ can be calculated as

$$
R_{i}=\left(\frac{d I_{D}}{d V_{D_{i}}}\right)^{-1}=\frac{1}{N K\left(V_{D_{i}}-V_{T}\right)}
$$

and the curvature as

$$
\gamma_{i}=\frac{d^{2} I_{D} / d V_{D_{i}}^{2}}{d I_{D} / d V_{D_{i}}}=\frac{1}{V_{D_{i}}-V_{T}} .
$$

Simplifying (1) by considering $R_{s, t o t} \ll R_{i}$, the zero-bias $\beta_{\text {opt }}$ is found as

$$
\beta_{\text {opt }}=\frac{1}{2} R_{i} \gamma_{i}=\frac{1}{2 N K\left(V_{D_{i}}-V_{T}\right)^{2}} .
$$

The major observation from (2)-(7) is how $n_{s}$ affects $R_{i}, \gamma_{i}$, and $\beta_{\text {opt }}$ in a zero-bias $\left(V_{D_{i}}=0 \mathrm{~V}\right)$ SSD with $R_{s, t o t}=0 \Omega$. First, $R_{i} \propto 1 / n_{s}$, and the same relationship holds for $\gamma_{i}$, resulting in $\beta_{\text {opt }} \propto 1 / n_{s}^{2}$. Similarly, $N E P_{\text {opt }} \propto 1 / n_{s}^{3 / 2}$. If the SSD is severely mismatched to the source $\left(R_{i} \gg 50 \Omega\right)$, $\beta_{50 \Omega} \propto 1 / n_{s}$, and $N E P_{50 \Omega} \propto n_{s}^{3 / 2}$. Notably, (2)-(7) predict the same relationships for $W$ as for $n_{s}$, assuming $C_{h}$ is independent of $W$.

Equations (2)-(7) are consistent with design dependencies that have been observed previously: Enhancing $C_{h}$, for example, by reducing $W_{h}$, can be expected to increase $\gamma_{i}$ and thus $\beta_{\text {opt }}$, which has been confirmed by MC simulations. ${ }^{13}$ It has further been reported that $\beta_{\text {opt }}$ in SSDs is enhanced when $W$ is decreased. ${ }^{5,6}$ The model derived above quantifies this effect.

The $n_{s^{-}}$and $W$-dependence suggested by (5)-(7) was compared to MC simulations. These simulations were carried out with a two-dimensional representation of an InAs SSD. ${ }^{14-16}$ The SSD design used in MC simulations was with $L=900 \mathrm{~nm}, W=35 \mathrm{~nm}$, and $n_{s}=1.5 \times 10^{10} \mathrm{~cm}^{-2} . W$ and $n_{s}$ were then varied independently. A single channel was simulated, and the resulting current was scaled to $Z=15 \mathrm{~nm}$ and multiplied by $N=43$ to obtain $I_{D}$. A uniform carrier concentration of $N_{D}=n_{s} / Z$ were used. No surface charge was placed on the channel sidewalls. $R_{s, t o t}$ was calculated at low $V_{D}$, and used to derive $V_{D_{i}}=V_{D}-I_{D}$. For every $n_{s}$ and $W$, the I-V curve was simulated for $V_{D} \in[-0.15,0.15] \mathrm{V}$. By fitting a 5th order polynomial to the simulated $I_{D}-V_{D_{i}}$ relationship, the intrinsic $\beta_{\text {opt }}$ at $V_{D}=0 \mathrm{~V}$ was calculated according to (7). For the modeled data, the same parameters were used as for simulations, with the addition of $\mu_{n}=28000 \mathrm{~cm}^{2} / \mathrm{V} \mathrm{s}$, as found from MC simulations. ${ }^{17} C_{h}$ was found as $C_{h}=\varepsilon_{0} Z / W_{h}$, where $\varepsilon_{0}$ is the permittivity of vacuum.

Fig. 2 shows $\beta_{\text {opt }}$ as acquired from MC simulations and the model in (4)-(7) for different $n_{s}$. The inset demonstrates a plot of $R_{i}$ and $\gamma_{i}$ as extracted from MC simulations compared to modeled values. The model accurately predicts the variation of $\beta_{\text {opt }}$. Also $R_{i}$ and $\gamma_{i}$ are modeled accurately. The $W$-dependence of $\beta_{o p t}, R_{i}$, and $\gamma_{i}$ is presented in Fig. 3, again comparing results from MC simulations to the model. Also for $W$, the modeled dependencies match the simulated data. Thus, the MC simulations confirm the SSD model in (7) for the intrinsic $\beta_{\text {opt }}$.

To experimentally investigate the influence of doping, InAs/ $/ \mathrm{Al}_{80} \mathrm{Ga}_{20} \mathrm{Sb}$ heterostructures with different $\delta$-doping

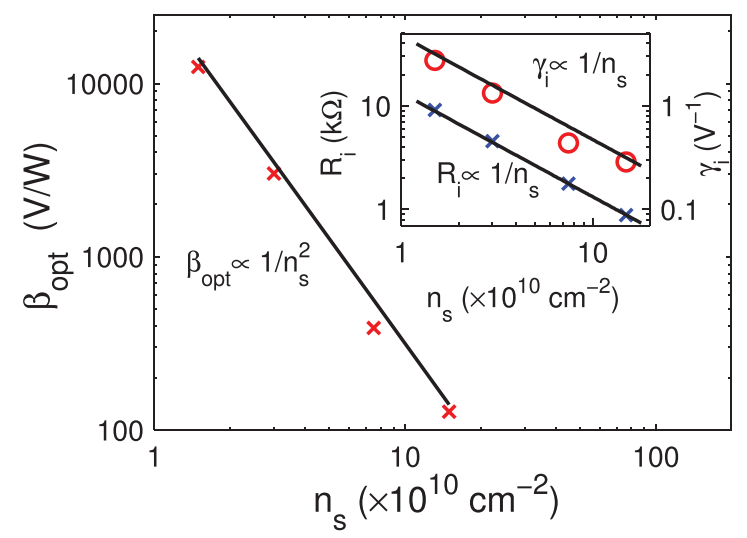

FIG. 2. Intrinsic $\beta_{\text {opt }}$ as derived from Monte Carlo simulations (crosses) and the model (solid line) as a function of $n_{s}$. The inset shows simulated $R_{\mathrm{i}}$ (crosses) and $\gamma_{\mathrm{i}}$ (circles), and the modeled counterparts (solid lines). 


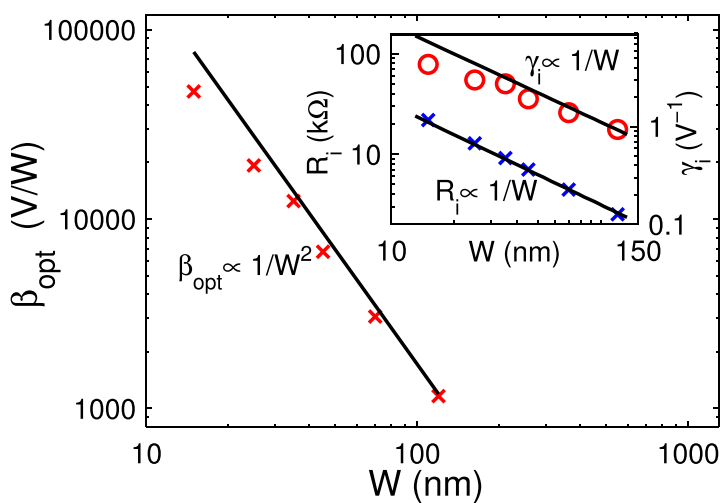

FIG. 3. Intrinsic $\beta_{\mathrm{opt}}$ as derived from Monte Carlo simulations (crosses) and the model (solid line) as a function of $W$. The inset shows simulated $R_{\mathrm{i}}$ (crosses) and $\gamma_{\mathrm{i}}$ (circles), and the modeled counterparts (solid lines).

were grown by molecular beam epitaxy. ${ }^{6}$ Similar structures have been used in InAs/AlSb high-electron-mobility transistors. ${ }^{18} \mathrm{An}$ AlSb metamorphic buffer was grown on a GaAs substrate. On top of the buffer, a $15 \mathrm{~nm}$ thick InAs/ $\mathrm{Al}_{80} \mathrm{Ga}_{20} \mathrm{Sb}$ quantum well was formed. The doping was placed in the $\mathrm{Al}_{80} \mathrm{Ga}_{20} \mathrm{Sb}$ barrier, $50 \AA$ above the InAs channel. To allow formation of low-resistivity ohmic contacts in subsequent device processing, a highly doped InAs cap layer was grown on top of the structure. In the active area, i.e., the area of the trenches, the cap layer was etched away. After the trench etch, the devices were passivated with silicon nitride using plasma enhanced chemical vapor deposition carried out at room temperature. Details in the fabrication process has been are given elsewhere. ${ }^{11}$

Two samples with different $\delta$-doping were used in SSD fabrication, one low-doped (i.e., non-intentionally doped, $\left.\delta=0 \mathrm{~cm}^{-2}\right)$ and one high-doped $\left(\delta=1 \times 10^{12} \mathrm{~cm}^{-2}\right)$. Hall measurements are shown in Table I, both for unprocessed samples and samples where the cap was etched and the surface passivated (similar to the active area of the SSDs). The etched Hall structure was processed on the same chip used in SSD fabrication. The low-doped sample showed a significant $n_{\mathrm{s}}$, in line with what have been reported for similar structures. ${ }^{19} n_{s}$ was higher in the etched than in the as-grown structures, especially in the low-doped sample where $n_{s}$ went from $0.61 \times 10^{12}$ to $1.5 \times 10^{12} \mathrm{~cm}^{2}$. As expected, the low-doped sample showed a higher $\mu_{n}$ than the high-doped sample. ${ }^{20}$

In Figs. 4-6, RF measurements of the InAs SSD detectors are compared to modeled counterparts. Zero-bias RF measurements of $\beta_{\text {opt }}$ were performed on-wafer at $50 \mathrm{GHz}{ }^{6}$ $N E P_{\text {opt }}$ and $N E P_{50 \Omega}$ was found from (2) using DC measurements of $R_{i}+R_{s, t o t}{ }^{6}$ The measurements on the low-doped

TABLE I. Sheet resistance $\left(R_{s h}\right)$, carrier concentration $\left(n_{\mathrm{s}}\right)$, and mobility $\left(\mu_{n}\right)$ from Hall measurements for the low- and high-doped samples used for InAs SSD fabrication.

\begin{tabular}{lcccc}
\hline \hline$\delta$-doping & & $\begin{array}{c}R_{\mathrm{sh}} \\
(\Omega / \mathrm{sq})\end{array}$ & $\begin{array}{c}n_{s} \\
\left(\times 10^{12} \mathrm{~cm}^{-2}\right)\end{array}$ & $\begin{array}{c}\mu_{n} \\
\left(\mathrm{~cm}^{2} / \mathrm{V} \mathrm{s}\right)\end{array}$ \\
\hline $0 \mathrm{~cm}^{-2}$ & As-grown & 435 & 0.61 & 23400 \\
& Etched and passivated & 164 & 1.5 & 26000 \\
$1 \times 10^{12} \mathrm{~cm}^{-2}$ & As-grown & 156 & 2.2 & 18300 \\
& Etched and passivated & 136 & 2.7 & 17000 \\
\hline \hline
\end{tabular}

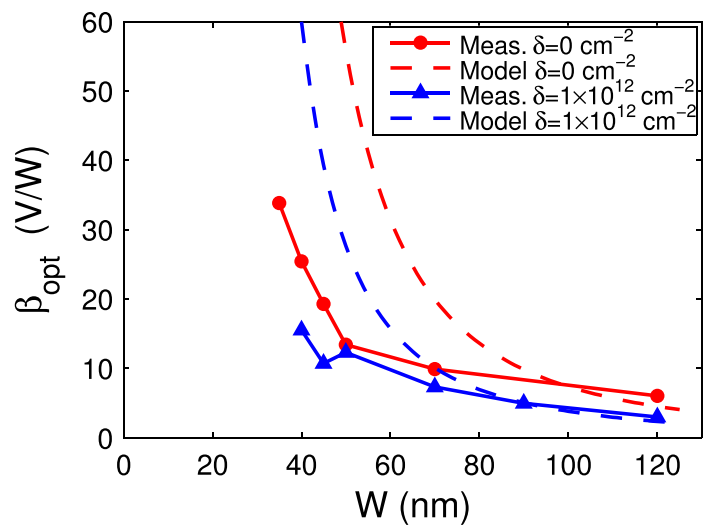

FIG. 4. Measured $\beta_{\mathrm{opt}}$ in low-doped and high-doped samples compared to modeled values.

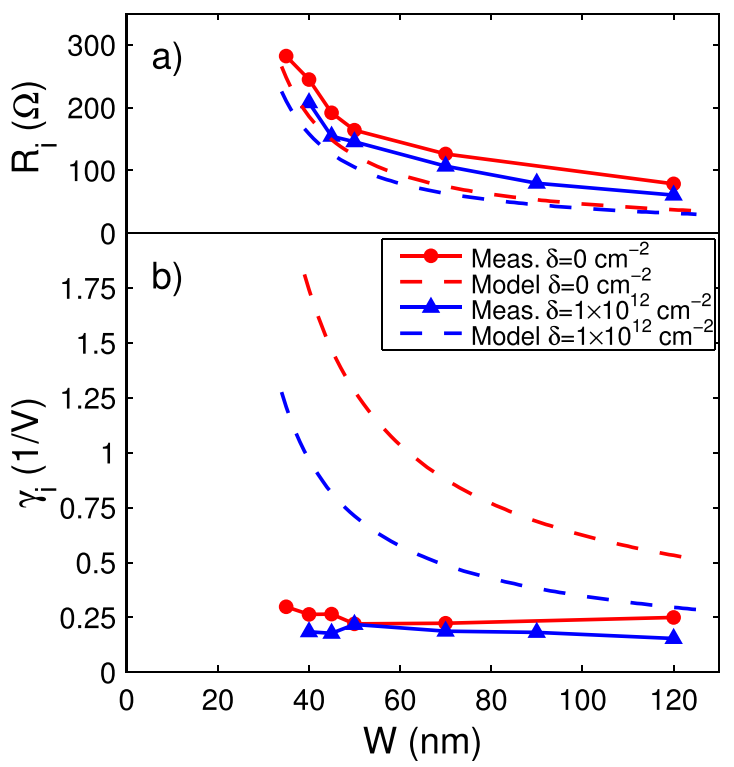

FIG. 5. (a) Measured $R_{i}$ and (b) $\gamma_{\mathrm{i}}$ in the low-doped and high-doped samples compared to modeled counterparts.

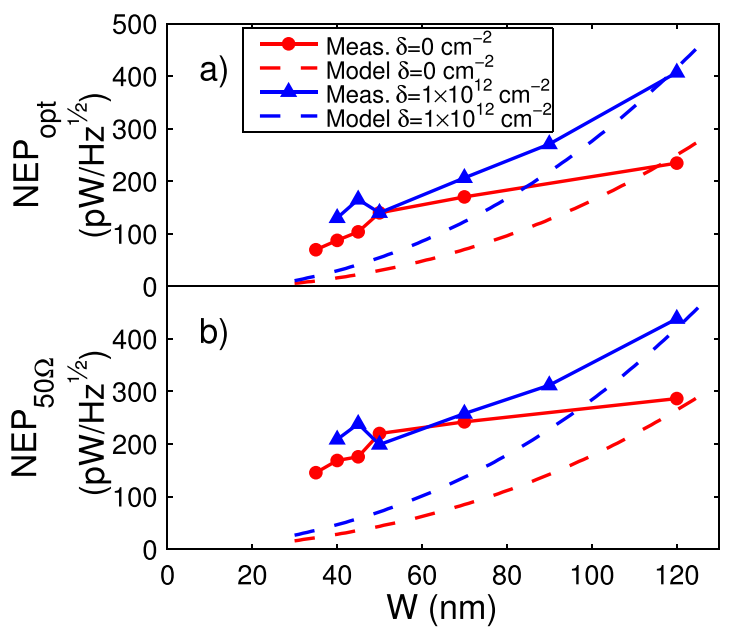

FIG. 6. (a) Estimated $N E P_{\text {opt }}$ and (b) $N E P_{50 \Omega}$ at $50 \mathrm{GHz}$ for the low-doped and high-doped samples compared to modeled counterparts.

sample has been published partly elsewhere. ${ }^{6}$ Modeled values were derived from (1)-(6) using measured $\mu_{n}$ and $n_{s}$, with the following considerations: $R_{s, t o t}$ was found by 
recognizing that $R_{s 2}=R_{i} W_{v} / L$. Further, $R_{s a}+R_{d a}$ was measured to $40 \Omega$ and $27 \Omega$ for the low- and high-doped samples, respectively, in structures without trenches (ignoring possible current-crowding effects at channel entrances). $2 C_{h}$ was calculated from the capacitance to ground in a coplanar transmission line with the dimensions $W, W_{h}$, and $L$ and the relative dielectric constant of AlSb $\varepsilon_{A l S b}=10.9 .^{21,22}$ The only adjustment of the model to measurements was that $W$ was replaced with an efficient channel width $W_{\text {eff }}$ $=W-20 \mathrm{~nm}$ to reproduce the measured $W$-dependence of $R_{i}{ }^{5}$ Consequently, $W_{h}$ was replaced with an efficient trench width $W_{h, \text { eff }}=W_{h}+20 \mathrm{~nm}$.

In Fig. $4, \beta_{\text {opt }}$ versus $W$ for the two samples is presented. As has been reported previously, ${ }^{5,6}$ reducing $W$ increases $\beta_{\text {opt. }}$ More importantly, Fig. 4 shows that the low-doped sample demonstrates a higher $\beta_{\text {opt }}$ than the high-doped samples. For $W=40 \mathrm{~nm}$, the smallest $W$ for which SSDs from both samples are available, the measurements exhibit a factor of $1.6 \times$ difference in $\beta_{\text {opt }}$ between the low- and high-doped sample, while the model predicts a factor of $2 \times$ difference. The model also overestimates $\beta_{\text {opt }}$ for small $W$. However, the model captures the relative dependencies well for both $n_{s}$ and $W$.

To further analyze how $\beta_{\text {opt }}$ is affected by $n_{s}, R_{i}$, and $\gamma_{i}$ are plotted in Fig. 5. $R_{\mathrm{i}}$ was derived from DC measurements and $\gamma_{i}$ from $\beta_{\text {opt }}$ using (1). $R_{i}$ is fairly similar in both samples. The low-doped sample shows a slightly higher $R_{\mathrm{i}}$ than the high-doped sample. The same trend was seen for $R_{s h}$ in Table I, where $R_{s h}$ was similar for all etched samples. From (5), $R_{i} \propto 1 / \mu_{n} n_{s}$ is expected. While $n_{s}$ is lower in the lowdoped sample, $\mu_{n}$ is higher, making $R_{s h}$ and thus also $R_{i}$ similar in both samples. The model which uses measured $n_{s}$ and $\mu_{n}$ reproduces $R_{i}$ fairly accurately.

While both samples exhibit similar $R_{i}$, the low-doped sample shows a significantly higher $\gamma_{i}$. This is explained by (6), where $\gamma_{i} \propto 1 / n_{s}$, and independent of variations in $\mu_{n}$. Thus, a larger difference in $\gamma_{i}$ than $R_{i}$ between samples could be expected. From the model and measured $n_{s}$, a factor $1.8 \times$ greater $\gamma_{i}$ could be expected in the low-doped sample than in the high-doped sample, for all $W$. In Fig. 5(b), for $W=40 \mathrm{~nm}$, the difference in $\gamma_{i}$ is a factor $1.4 \times$, in good agreement with the model. The model shows considerably stronger $W$-dependence of $\gamma_{i}$ than measurements, and overestimates $\gamma_{i}$ which led to also $\beta_{\text {opt }}$ being overestimated for small $W$ in Fig. 4. Measurements exhibit the predicted trend qualitatively: InAs SSDs from the low- and high-doped samples with $W=35 \mathrm{~nm}$ and $W=40 \mathrm{~nm}$, respectively, showed $20 \%$ higher $\gamma_{i}$ compared to devices with $W=120 \mathrm{~nm}$.

Fig. 6 demonstrates the effect on $N E P_{\text {opt }}$ and $N E P_{50 \Omega}$ for varying $\delta$-doping. As has been shown elsewhere, ${ }^{6} N E P_{\text {opt }}$ and $N E P_{50 \Omega}$ decrease when reducing $W$. Further, the lowdoped sample exhibited both a lower $N E P_{\text {opt }}$ and $N E P_{50 \Omega}$ than the high-doped sample. The model underestimates both $N E P_{o p t}$ and $N E P_{50 \Omega}$ for small $W$ due to the overestimated $\beta_{\text {opt }}$. However, the difference in $N E P$ related to $\delta$-doping is well described by the model, which predicts a $46 \%$ lower $N E P_{\text {opt }}$ in the low-doped sample than in the high-doped sample. Comparing the low- and high-doped sample for $W=40 \mathrm{~nm}$, the former shows an $N E P_{\text {opt }}$ reduced with $33 \%$ $\left(130 \mathrm{pW} / \mathrm{Hz}^{1 / 2}\right.$ and $87 \mathrm{pW} / \mathrm{Hz}^{1 / 2}$, respectively) whereas the model predicts a $46 \%$ reduction.
It was found from measurements of fabricated devices, model and MC simulations that lowering the $\delta$-doping is beneficial for SSD direct detector performance with respect to responsivity and NEP. Reducing $n_{s}$ increases $\gamma_{i}$, but also $R_{\mathrm{i}}$, provided $\mu_{n}$ is constant. High $R_{\mathrm{i}}$ requires a more complex matching network and as shown in (2), $R_{i}$ contributes to noise, while $\gamma_{\mathrm{i}}$ does not. Increasing $\gamma_{\mathrm{i}}$ will therefore always improve both responsivity and NEP.

Published SSDs have shown $\beta_{50 \Omega}<200 \mathrm{~V} / \mathrm{W}^{2,4-6}$ Since $\beta_{50 \Omega}=100 \gamma_{i}$ for $R_{i} \gg 50 \Omega$, all published SSDs have $\gamma_{i}<2 \mathrm{~V}^{-1}$. Zero-biased Schottky diodes reaches $\gamma_{i}$ close to their theoretical limit of $39.6 \mathrm{~V}^{-1}$. There is thus a large potential in increasing $\gamma_{i}$ in SSDs. As shown in Figs. 2-6, lowering the $\delta$-doping and thus $n_{s}$ is a powerful way to enhance $\gamma_{i}$.

We have studied how the level of $\delta$-doping in the InAs/ $\mathrm{Al}_{80} \mathrm{Ga}_{20} \mathrm{Sb}$ heterostructure affects the SSD direct detector performance. By treating the SSD as a double side-gated transistor, responsivity and NEP of the detector could be expressed as a function of design and material parameters $\mu_{n}$ and $n_{s}$. The voltage responsivity with an optimal power match was theoretically found to be proportional to $1 / n_{s}^{2}$. A similar relationship was found for the channel width. Monte Carlo simulations of InAs SSDs confirmed the model. When comparing to measurements of fabricated devices, the model reproduced the main features of both $\beta_{o p t}, N E P_{\text {opt }}$ and $N E P_{50 \Omega}$. The $n_{s}$-dependence showed good agreement between model and measurements. By a combination of theory, simulations, and measurements, it was shown that lowering the $\delta$-doping improves the performance of SSD direct detectors.

This work was sponsored by the European Commission FP7 through the ROOTHz Project ICT-2009-243845.

${ }^{1}$ A. M. Song, M. Missous, P. Omling, A. R. Peaker, L. Samuelson, and W. Seifert, Appl. Phys. Lett. 83, 1881 (2003).

${ }^{2}$ C. Balocco, S. R. Kasjoo, X. F. Lu, L. Q. Zhang, Y. Alimi, S. Winnerl, and A. M. Song, Appl. Phys. Lett. 98, 223501 (2011).

${ }^{3}$ J. Torres, P. Nouvel, A. Penot, L. Varani, P. Sangaré, B. Grimbert, M. Faucher, G. Ducournau, C. Gaquière, I. Iñiguez-de-la-Torre, J. Mateos, and T. Gonzalez, Semicond. Sci. Technol. 28, 125024 (2013).

${ }^{4}$ C. Balocco, M. Halsall, N. Q. Vinh, and A. M. Song, J. Phys. Condens. Matter 20, 384203 (2008).

${ }^{5}$ P. Sangaré, G. Ducournau, B. Grimbert, V. Brandli, M. Faucher, C. Gaquière, A. Íñiguez-de-la-Torre, I. Íñiguez-de-la-Torre, J. F. Millithaler, J. Mateos, and T. González, J. Appl. Phys. 113, 034305 (2013).

${ }^{6}$ A. Westlund, P. Sangaré, G. Ducournau, P.-A. Nilsson, C. Gaquière, L. Desplanque, X. Wallart, and J. Grahn, Appl. Phys. Lett. 103, 133504 (2013).

${ }^{7}$ J. L. Hesler and T. W. Crowe, in Proceedings of the 18th International Symposium on Space Terahertz Technology, Pasadena, CA, 2007.

${ }^{8}$ A. Iñiguez-de-la-Torre, I. Íñguez-de-la-Torre, J. Mateos, T. Gonzalez, P. Sangare', M. Faucher, B. Grimbert, V. Brandli, G. Ducournau, and C. Gaquière, J. Appl. Phys. 111, 113705 (2012).

${ }^{9}$ G. Farhi, E. Saracco, J. Beerens, D. Morris, S. A. Charlebois, and J.-P. Raskin, Solid. State. Electron. 51, 1245 (2007).

${ }^{10}$ M. Åberg, J. Saijets, A. Song, and M. Prunnila, Phys. Scr. T114, 123 (2004).

${ }^{11}$ A. Westlund, G. Moschetti, P.-A. Nilsson, J. Grahn, L. Desplanque, and X. Wallart, in Proceedings of the 2013 International Conference on Indium Phosphide and Related Materials, IEEE, Osaka, Japan, 2013.

${ }^{12}$ A. M. Cowley and H. O. Sorensen, IEEE Trans. Microwave Theory Tech. 14, 588 (1966).

${ }^{13}$ I. Iñiguez-de-la-Torre, J. Mateos, D. Pardo, A. M. Song, and T. González, Int. J. Numer. Modell. Electron. Networks Devices Fields 23, 301 (2009). 
${ }^{14}$ I. Iñiguez-de-la-Torre, H. Rodilla, J. Mateos, D. Pardo, A. M. Song, and T. González, J. Phys. Conf. Ser. 193, 012082 (2009).

${ }^{15}$ J. Mateos, B. G. Vasallo, D. Pardo, T. Gonzalez, J. Galloo, S. Bollaert, Y. Roelens, and A. Cappy, IEEE Trans. Electron Devices 50, 1897 (2003).

${ }^{16}$ J. Mateos, B. G. Vasallo, D. Pardo, and T. Gonzalez, Appl. Phys. Lett. 86, 212103 (2005).

${ }^{17}$ H. Rodilla, T. González, D. Pardo, and J. Mateos, J. Appl. Phys. 105, 113705 (2009).
${ }^{18}$ G. Moschetti, P.-A. Nilsson, A. Hallen, L. Desplanque, X. Wallart, and J. Grahn, IEEE Electron Device Lett. 33, 510 (2012).

${ }^{19}$ G. Tuttle, H. Kroemer, and J. H. English, J. Appl. Phys. 65, 5239 (1989).

${ }^{20}$ B. R. Bennett, J. B. Boos, M. G. Ancona, N. A. Papanicolaou, G. A. Cooke, and H. Kheyrandish, J. Electron. Mater. 36, 99 (2007).

${ }^{21}$ G. Ghione and C. Naldi, Electron. Lett. 20, 179 (1984).

${ }^{22}$ K. Seeger and E. Schonherr, Semicond. Sci. Technol. 6, 301 (1991). 